Políticas públicas y derecho a la educación de niños, niñas y adolescentes extranjeros en Chile

Publics Policies and right to education from foreign children and adolescents in Chile

\title{
Volumen 17, Número 1
}

Enero-Abril

pp. $1-19$

Carolina Jorquera-Martínez

Revista indizada en REDALYC, $\underline{\text { SCIELO }}$

Revista distribuida en las bases de datos:

LATINDEX, DOAJ, REDIB, IRESIE, CLASE, DIALNET, SHERPA/ROMEO, QUALIS-CAPES, MIAR

Revista registrada en los directorios:

ULRICH'S, REDIE, RINACE, OEI, MAESTROTECA, PREAL, CLACSO 


\title{
Políticas públicas y derecho a la educación de niños, niñas y adolescentes extranjeros en Chile
}

\author{
Publics Policies and right to education from foreign children and adolescents in Chile
}

\section{Carolina Jorquera-Martínez ${ }^{1}$}

Resumen: El objetivo del presente artículo es evaluar las políticas públicas locales, desde un enfoque cualitativo usando análisis documental, considerando el ajuste de éstos con la incorporación al sistema escolar de niños, niñas y adolescentes (NNA) extranjeros, tomando en cuenta que el reconocimiento de la diversidad sociocultural es parte de las dimensiones de una educación de calidad. Los resultados muestran que los dispositivos que regulan la educación en Chile consideran de manera global la no discriminación y el acceso universal a esta. Sin embargo, faltan normativas explícitas que medien la incorporación de estudiantes extranjeros que consideren ando sus necesidades administrativas y pedagógicas. La principal recomendación a la luz de los hallazgos tiene relación con explicitar esta dimensión en procedimientos, instancias y recursos que permitan una positiva integración de este tipo de estudiantes, para asegurar el logro de sus aprendizajes integrales.

Palabras clave: derecho a la educación, migración, políticas públicas

\begin{abstract}
The objective of the present article is to evaluate from a qualitative approach-by documental analysisthe adjustment of the current educational public policies in Chile, the incorporation to the school system of foreign boys, girls and teenagers (NNA). We are assuming that the recognition of sociocultural diversity is part of the dimensions in order to get an education quality. The results show that the devices that regulate education in Chile globally consider no discrimination and universal access to it. However, there is a lack of explicit regulations that can mediate the incorporation of foreign students considering their administrative and educational needs. The main recommendation, given the discoveries, it's related to explicitly stating this dimension, generating procedures, instances and resources that allow a positive integration of this kind of students, ensuring the achievement of their comprehensive learnings.
\end{abstract}

Keywords: right to education, migration, public policies

\footnotetext{
1 Académica de la Escuela de Psicología de la Universidad de Santiago de Chile. Psicóloga educacional.
}

Dirección electrónica: carolina.jorquera@usach.cl

Artículo recibido: $1^{\circ}$ de marzo, 2016

Enviado a corrección: 6 de setiembre, 2016

Aprobado: 21 de noviembre, 2016 


\section{Introducción ${ }^{2}$}

La educación no es tan solo una herramienta del desarrollo personal y económico, sino que es un derecho explícito en numerosos instrumentos jurídicos supranacionales desde su inclusión en la Declaración Universal de Derechos Humanos, en función de ser el medio privilegiado para la generación de aprendizajes fundamentales para cada uno de los miembros de una sociedad, de manera que cumpla con sus metas de desarrollo personal, el de su comunidad y la consecución de los otros derechos humanos.

En ese sentido, es importante considerar que todo niño, niña y adolescente -sin discriminación alguna- debe tener acceso a educarse, lo que implica para los Estados asegurar una oferta educativa que permita la participación universal dentro de los procesos de escolarización. Sin embargo, cabe destacar que dicha característica no es la única dimensión para evaluar el cumplimiento de dicho derecho. Es necesario considerar que la educación provista sea acorde con las necesidades de aprendizaje, pertinente con las características culturales de los educandos y respetuosa de las libertades fundamentales, teniendo como eje rector la dignidad humana, tanto en los contenidos trabajados en las aulas de clase, como en las prácticas promovidas por las diversas formas que puede tomar la escolarización, Lo anterior, ha sido explicitado en las observaciones 11 y 13 del Comité de derechos económicos, sociales y culturales, siendo incluido en las instancias de evaluación de sistemas y estrategias educativas en sus distintos niveles (Tomasevsky, 2004).

Cada Estado, como parte de sus obligaciones, debería generar las condiciones para el cumplimiento del derecho a la educación, considerando acciones, presupuestos y normativas reguladoras que permitan proteger, respetar y promover dicho derecho de niños, niñas y adolescentes. En este ámbito, las características estructurales del sistema escolar de Chile han sido evaluadas positivamente en cuanto al acceso a la educación; sin embargo, se ha considerado de manera negativa la alta segmentación que este propende, reconociendo como fuentes de desigualdad aquellas propias del origen de los estudiantes, incluyendo su origen étnico (López, 2014).

En el presente trabajo, se ha tomado en cuenta el caso específico de los educandos que tienen origen extranjero, ya sea inmigrantes o hijos de inmigrantes nacidos en el país. Lo anterior debido a que la realidad migratoria de Chile es un fenómeno que ha sufrido un brusco cambio en sus lógicas históricas. El aumento en los flujos migratorios en las últimas décadas genera una brecha importante entre las características de la institucionalidad

\footnotetext{
${ }^{2}$ Investigación financiada por la Dirección de Investigación, Ciencia y Tecnología (DICYT) de la Universidad de
} Santiago de Chile 
escolar vigente y las necesidades propias de ese creciente grupo de población. Dicha brecha ha sido evaluada con preocupación por distintos actores, pues la normativa legal vigente se encuentra obsoleta, lo que agudiza la segmentación y desigualdad ya presente en el sistema. Las normativas y procedimientos del sistema educativo no responden de manera adecuada a los desafíos de un mundo con mayor movilidad territorial de los sujetos, generando vacíos que conllevan a la vulneración de los derechos de las personas (Lagos y Rojas, 2014; Olea, 2013).

La incorporación de este tipo de estudiante al interior de las escuelas tiene que ver con lo que los estudios de la sociología de la infancia denominan la herencia de la condición migrante, y que tiene relación con la compleja red de significados que asocian la nacionalidad a procesos de crianza y de origen familiar, siendo esta la base de reconocimiento de los niños (Pavez, 2012). En ese sentido, es sumamente relevante considerar el hecho de que la educación ha tenido tensiones históricas con los marcos referenciales que tienen los núcleos familiares, las cuales son explicativas de los resultados de éxito o fracaso escolar en función de la cercanía o lejanía que estos tengan con la cultura letrada. El capital social de origen, por tanto, configura las transacciones de saberes, prácticas culturales y significados, en general, que implica la escuela.

El Banco Mundial (2015) cifra la tasa de migración neta ${ }^{3}$ para nuestro país en 30000 en el periodo entre los años 2010-2014, siendo el único territorio de la región junto con Venezuela que tiene una tasa positiva; lo que implica que el número de personas que ingresa al país es mayor que las que salen de este. Fuentes de datos en el mismo tópico señalan que por cada 100 inmigrantes residiendo hoy en el país, hay 140 chilenos, nacidos en el país, que viven en tierras extranjeras (Agar, 2012).

Las cifras de estimación de la población a partir del Censo del 2012 que ha hecho el Departamento de Extranjería y Migración han mostrado que dicho fenómeno en Chile no tan solo ha aumentado un $84 \%$ en los últimos 10 años, sino también ha modificado la composición de la población extranjera que llega al país. La inmigración actual puede caracterizarse como más variada que en épocas anteriores donde se producía movilidad centralmente entre países vecinos. Actualmente, se han sumado personas de distintos países de Latinoamérica, no solo los fronterizos, e inclusive de otros continentes (Norambuena, 2013).

\footnotetext{
${ }^{3}$ Total neto de personas que migraron durante el periodo: cantidad total de inmigrantes menos la cantidad anual de emigrantes.
} 
Una característica fundamental para poder comprender la relación entre las políticas públicas del sistema educativo en Chile, el aseguramiento y garantía de los derechos humanos y la situación de infantes y adolescentes de origen extranjero, es el perfil feminizado de la migración en nuestro país. Las cifras muestran que, tal como en el resto de la región, la mayor parte de las personas que ingresan a nuestro país son mujeres, quienes tienen entre sus principales preocupaciones reunir nuevamente a su familia, así como llevar adelante proyectos familiares en el país. Los Estados deben procurar proteger los derechos de NNA y sus madres, generando políticas donde dichos elementos estén por sobre la preocupación acerca de la regulación de la situación migratoria de las mujeres; sin embargo, situación que no está asegurada dentro de las disposiciones legales vigentes. (Lagos y Rojas, 2014).

Dentro de estas políticas que el Estado debería desarrollar, la importancia antes referida a la educación hace de esta un foco crítico para el aseguramiento del desarrollo de NNA, permitiendo a su vez -a partir del rol sociocomunitario de las escuelas- aportar en la promoción de derechos y de desarrollo, impactando en aquellos que rodean al estudiantado. La acción estatal en este caso incluye los distintos tipos de dispositivos de gestión y actores encargados de concretar en la vida cotidiana de las escuelas el derecho a la educación con todas las dimensiones involucradas. Lo anterior implica considerar acciones a nivel central configuradas por todas aquellas disposiciones que enmarcan la acción de todo el sistema escolar a nivel curricular y de organizaciones escolares- y acciones de mayor especificidad territorial y administrativa, como lo son las disposiciones de los departamentos municipales de educación o las disposiciones educativas que plantee cada uno de los establecimientos.

En este marco, el presente artículo trabaja en torno a la siguiente pregunta de investigación: ¿Cuál es la adecuación de los dispositivos de la política pública en pro del aseguramiento del derecho a la educación de NNA de origen migrante? Teniendo consecuentemente como objetivos: caracterizar los dispositivos de la política pública evaluando el ajuste que estos tengan en función al aseguramiento del derecho a la educación de dicho grupo de la población. Para el logro de lo anterior, se rastrean rasgos que faciliten la incorporación del estudiantado de origen migrante dentro de los documentos regulatorios de la educación en Chile, a nivel nacional y a nivel municipal, dentro de la provincia de Santiago. 


\section{Metodología}

El desarrollo del presente trabajo se enmarca en el paradigma cualitativo de la investigación, asumiendo la tarea de la deconstrucción y reconstrucción del conocimiento social a partir del análisis de distintos tipos de textos en una aproximación científica (Mejía, 2004). Como técnica se ha aplicado el análisis documental, realizándose análisis de contenido a dos tipos de texto:

Documentos oficiales que dan cuenta de la política pública educativa a nivel nacional, buscando acciones que desde el Estado puedan asegurar el derecho a la educación de NNA de origen migrante, tanto en las disposiciones curriculares como en las políticas asociadas al ámbito de convivencia escolar realizadas por el Ministerio de Educación de nuestro país. Los documentos revisados fueron: Ley 20.370 (Ministerio de Educación, 17 de setiembre, 2011); Oficio Curricular 1179 (Depto. Extranjería y Migración, 2003); Decreto 439/2012 (Ministerio de Educación, 2011); Decreto 254/2009; Política Nacional de convivencia escolar (Chile, 2015), Ley de Violencia Escolar 20536 (Ministerio de Educación, 8 de setiembre, 2011) y Otros indicadores de calidad educativa (Ministerio de Educación, 2014) ${ }^{4}$, que orienta con respecto a aquellos rasgos de la escuela que se incluyen en la evaluación de los establecimientos del país y que complementa las pruebas estandarizadas asociadas a la cobertura curricular de asignaturas claves al interior de los distintos ciclos de formación que incluye el sistema escolar.

Para representar un nivel distinto de regulación del sistema educativo --más cercano a la experiencia de las personas involucradas- se analizó un segundo tipo de documento: los planes anuales de educación (Padem) del 2015 de las comunas de la provincia de Santiago publicados en sitios de internet oficiales de cada municipio; el corpus documental en este caso asciende a 19 planes de educación.

En todos los casos se consideraron las alusiones explícitas a la migración como rasgo de diversidad de los NNA atendidos por el sistema educativo.

\footnotetext{
${ }^{4}$ El nombre de Otros Indicadores de Calidad Educativa hace referencia a la integración en la evaluación de los establecimientos educacionales del país de aspectos complementarios a los tradicionalmente medidos en pruebas estandarizadas asociadas a la cobertura curricular en algunos sectores del curriculum, y que se hace por medio de la Prueba SIMCE desde el año 1988 en Chile
} 


\section{Antecedentes contextuales de la incorporación de estudiantado extranjero en el sistema escolar chileno}

En primer lugar, se hace necesario describir de manera breve el sistema educacional chileno, dando cuenta de problemáticas que son transversales a los establecimientos, comunidades y actores involucrados en los procesos educativos y que están en la base del impulso de una serie de medidas destinadas a reformar la educación en el país y que han sido parte del movimiento social pro educación, que es un actor social relevante con fuerte presencia desde fines de la primera década del 2000.

La escolaridad es obligatoria en Chile (desde el 2002) durante doce años consecutivos de formación, lo que incluye los periodos de enseñanza básica (primaria) y media (secundaria). Existe un acceso casi universal en ambos niveles formativos teniendo además tasas aceptables de eficiencia interna en términos de retención y aprobación. Los establecimientos educacionales en Chile financiados por el Estado pueden ser administrados por los municipios o por particulares, ya sea como personas naturales o jurídicas, pudiendo estos últimos cobrar a padres y apoderados, cuotas de mensualidad para el funcionamiento de la escuela. Este tipo de estructura del sistema ha derivado en una alta segmentación correlacionando el nivel socioeconómico de las familias con la calidad de educación recibida por niños, niñas y adolescentes; situación que ha sido evaluada como una vulneración con respecto al derecho a la educación en Chile. Una última característica a consignar del sistema educativo tiene relación con las prácticas de selección de estudiantes que llevan a cabo los establecimientos, lo que también atenta contra el derecho humano antes citado (López, 2014).

\subsection{Derecho a la educación y situación migratoria de NNA en Chile}

Uno de los primeros antecedentes por considerar en este caso es el hecho de que los Estados están obligados a proteger y garantizar los derechos humanos de todos quienes se encuentren bajo su jurisdicción, independiente de su situación migratoria. Lo anterior es coherente con el principio de no discriminación y la no admisión de restricciones que deben tener los derechos humanos (OEA, 1988) ${ }^{5}$.

Considerando los dispositivos normativos de derechos humanos, cabe destacar que Chile ha ratificado los siguientes instrumentos asociados con la situación de migración: Convención sobre estatuto de los refugiados (ratificada en1972), Convención internacional

\footnotetext{
${ }^{5}$ Suscrito por Chile el 5 de junio de 2001, ratificación en proceso.
} 
sobre la eliminación de todas las formas de discriminación racial (ratificada en 1972), Convención internacional sobre la eliminación de todas las formas de discriminación contra la mujer (ratificada en 1989), Convención internacional sobre los derechos del niño (ratificada en 1990), Convención internacional sobre la protección de los derechos de todos los trabajadores migratorios y de sus familiares migrantes (ratificado en 2005), (López, 2014).

Asimismo, y tal como sucede con respecto a otros ámbitos de los derechos humanos, la Constitución Política de la República de Chile explicita la adscripción que el cuerpo normativo de la nación hace a las convenciones de este tipo de derechos; entendiéndose que dichos dispositivos son válidos y exigibles para las distintas áreas de aplicación dentro del funcionamiento del país.

La regulación de la situación migratoria, por lo tanto, debiese ser un aspecto que no puede ser considerado un requisito para el acceso de ningún niño, niña o adolescente a la educación. Por este motivo, debe considerarse que tanto las naciones emisoras como receptoras de migrantes deben velar por adecuadas condiciones de vida de las personas especialmente en el acceso a derechos básicos para una vida digna (Agar, 2012). Sin embargo, se han observado casos en los cuales se niegan las matrículas a quienes no han regularizado dicha situación, se han generado problemáticas en relación con el registro de sus calificaciones y certificación de sus avances y se reportan prácticas discriminatorias por parte de distintos actores educativos (Chile, Instituto Nacional de Derechos Humanos, 2012; Pavez, 2012, 2013).

En caso de las personas migrantes, las recomendaciones que se le han hecho al Estado de Chile tienen relación con modificar la ley de migración y realizar una serie de acciones que permitan asegurar la no discriminación ni estigmatización de las personas en las distintas esferas de la vida social, incluyendo la realización de campañas de promoción y formación de distintos agentes involucrados, entre los cuales se cuentan los docentes (Chile, Instituto Nacional de Derechos Humanos, 2012). En el último año, distintas iniciativas en Chile han asumido la tarea de sensibilizar por una nueva ley de extranjería que sea funcional a las características de la movilidad de las personas que se vivencia actualmente en el mundo y que incluya la multidimensionalidad del fenómeno migrante. 


\section{Presencia de la problemática migrante en las políticas públicas: nivel gobierno central y municipal}

A partir del análisis de contenido, se revelaron aquellos elementos de la política pública a nivel central y municipal que están asociados a la garantía del derecho a la educación a niños, niñas y adolescentes extranjeros que viven en Chile. Para definir acciones, estrategias y objetivos asociados a esta temática, se definieron como facilitadores de la incorporación del estudiantado migrante cualquiera de estos elementos que estuviera asociado a principios de no discriminación o interculturalidad. Lo anterior debido al cariz emergente de dicho fenómeno en el sistema escolar, así como a la ausencia de estructuras normativas derivadas de las falencias de la ley de extranjería que habían sido pesquisadas en el rastreo de antecedentes teóricos y normativos de la presente investigación.

Debido a la importancia que tienen los procesos de concreción curricular, los resultados se presentan siguiendo la lógica de dichos procesos, desde los elementos propios del curriculum prescrito a nivel central, hasta los elementos del nivel de gestión municipal rastreando en diversos reglamentos, normativas y programas que regulan el sistema educativo en Chile.

\subsection{Nivel de gestión en sistema nacional:}

El instrumento que estructura y regula la educación en Chile es la Ley General de Educación, vigente desde el 2009. En ella se define el sentido y las características que debiera tener el proceso educativo del país, considerando los aportes de los distintos actores vinculados con el sistema. En su artículo 2 se define la educación como un proceso permanente con la finalidad de desarrollo integral. En términos éticos declara explícitamente aquellos elementos que la sustentan, así como la finalidad del proceso de escolarización:

(...) Se enmarca en el respeto y valoración de los derechos humanos y de las libertades fundamentales, de la diversidad multicultural y de la paz, y de nuestra identidad nacional, capacitando a las personas para conducir su vida en forma plena, para convivir y participar en forma responsable, tolerante, solidaria, democrática y activa en la comunidad, y para trabajar y contribuir al desarrollo del país. (Ministerio de Educación, 17 de setiembre, 2011, p. 1)

Desde su definición, entonces, encontramos un proceso que se piensa como una instancia para un desarrollo integral que atiende diversas necesidades y aristas del ser 
humano. Asimismo, explicita el respeto y valoración de los derechos humanos y la diversidad multicultural, lo que implicaría la definición de un sistema educativo que debiera atender también la pluralidad migratoria como parte de su quehacer.

La apertura a esta variedad cultural es reforzada en el artículo 3 del mismo cuerpo legal al declarar la educación sustentada en derechos y tratados internacionales, especialmente aquellos que se refieren al derecho a la educación. Asimismo, en ese mismo artículo enuncia los once principios que rigen la educación en Chile. De estos, existen siete que tienen directa relación con el reconocimiento a la identidad cultural y la no discriminación al interior de las escuelas: universalidad y educación permanente, calidad de la educación -independiente de las características y circunstancias de las personas-, equidad, diversidad, flexibilidad, integración e interculturalidad.

Finalmente, es necesario destacar que se explicita claramente que "es deber del Estado velar por la igualdad de oportunidades y la inclusión educativa, promoviendo especialmente que se reduzcan las desigualdades derivadas de circunstancias económicas, sociales, étnicas, de género o territoriales, entre otras" (Ministerio de Educación, 17 de setiembre, 2011, Artículo 4). Otro deber explícito del Estado en la ley es "promover el estudio y conocimiento de los derechos esenciales que emanan de la naturaleza humana; fomentar una cultura de la paz y de la no discriminación arbitraria (...) y la diversidad cultural de la Nación" (Ministerio de Educación, 17 de setiembre, 2011, Artículo 5).

De igual forma, en la Ley General de Educación se explicita que los alumnos y alumnas tienen derecho a recibir una educación integral que no los discrimine arbitrariamente, que sean atendidas oportunamente sus necesidades diferenciales para alcanzar las metas formativas, y que el proceso educativo sea realizado en un ambiente tolerante y de respeto mutuo, siendo promovidos y evaluados por un sistema transparente (Ministerio de Educación, 17 de setiembre, 2011, Artículo 10).

Todos los artículos antes citados ayudan a configurar el mandato que desde la normativa legal se hace a las escuelas para que acojan a niños, niñas y adolescentes de origen extranjero considerando sus características culturales como variable mediadora y no un obstáculo para su aprendizaje. Cada uno de los establecimientos debiera entonces asegurar un clima escolar y procedimientos no discriminatorios para ellos. Sin embargo, cabe destacar que las disposiciones son genéricas y que responden a una amplia definición de diversidad cultural sin que se especifiquen sus rasgos y dimensiones, no 
considerando las lógicas distintas en la construcción de distintos modos de configurar la alteridad.

Dentro de las orientaciones de tipo administrativo que rigen a nivel del sistema educacional completo existe la disposición administrativa que otorga la posibilidad de acceder a una visa de estudiante por cualquier niño, niña y adolescente matriculado en establecimientos educacionales en cualquiera de los niveles que tiene el sistema en el país, independientemente de la situación migratoria de sus padres, madres y/o cuidadores (Depto. de Extranjería y Migración, 2003). Según datos solicitados a la dirección de extranjería, durante el 2014, se emitieron 15136 visas de este tipo de un total de 128 032, lo que representa el $11.8 \%$ del total de visas emitidas dicho año. En el caso de las permanencias definitivas de estudiantes, se otorgaron 4269 permisos de un total de 36 025, representando la misma proporción dentro del grupo.

En el 2005, se emite un oficio, desde la división jurídica del Ministerio de Educación, para instruir a los establecimientos sobre el ingreso, permanencia y ejercicio de los derechos de alumnos inmigrantes a las escuelas reconocidas por dicho organismo. En él se declara explícitamente el deber del Estado para la implementación de mecanismos que permitan el ingreso y permanencia de niños, niñas y jóvenes en igualdad de condiciones de los chilenos, cautelando que no existan discriminaciones arbitrarias del alumnado migrante. Se instruye explícitamente a las escuelas que deben otorgar facilidades para el ingreso al sistema escolar: lo anterior implica una matrícula provisoria bajo autorización de los departamentos provinciales solo con un documento que acredite edad y nombre del NNA, y en el caso de certificados de últimos estudios, la presentación de estos aún sin el trámite de legalización. Esta matrícula implica la subvención correspondiente al establecimiento por la asistencia del educando, cobertura de seguro escolar y el derecho a participar de todas las instancias formativas del establecimiento. El mismo oficio establece la obligatoriedad que en breve plazo el estudiante o la estudiante obtenga su permiso de residencia en condición de estudiante titular conforme con las normativas vigentes del Departamento de Extranjería y Migración del Ministerio del Interior.

Considerando aquellas disposiciones a nivel curricular y que definen los aprendizajes por alcanzar por los niños, niñas y adolescentes dentro de la escuela (Decreto 439/2012 para educación general básica, Decreto 254/2009 para enseñanza media), encontramos que a nivel de planes y programas aparece la diversidad cultural dentro de los objetivos fundamentales transversales en función de aprendizajes que permitan el respeto de los otros, sin especificar la fuente de dicha diversidad. En el caso de los espacios de corte 
instruccional, en los planes de asignatura de historia, geografía y ciencias sociales, coexisten menciones a esta diversidad cultural con la meta que tiene esta asignatura de preservar la identidad nacional, lo que genera una tensión importante en el diseño didáctico que no tiene orientaciones en su resolución.

Otra fuente de disposiciones por tomar en cuenta es aquella que tiene que ver con la generación de ambientes de los aprendizajes dentro de la escuela. Entre ellas podemos encontrar la Política de Convivencia Escolar, Ley de Violencia Escolar y los Otros Indicadores de Calidad Educativa. Todas estas disposiciones abordan la convivencia como un ámbito que debe "intencionarse" al interior de las escuelas, trabajando en torno a que cada una de ellas tenga un ambiente seguro, no discriminatorio y que permita a los NNA aprender a relacionarse positivamente con otros. Dichos principios y orientaciones facilitan la construcción de establecimientos que resguarden la diversidad cultural; sin embargo, en general, no hay disposiciones específicas que se refieran al estudiantado de origen migrante. Una excepción a lo anterior está en la actualización de la Política Nacional de Convivencia Escolar que se realizó en el 2015 y que indica explícitamente la necesidad de que los proyectos educativos institucionales de cada establecimiento deben basarse en principios de no discriminación, contando con protocolos claros de retención y resguardo de derechos de tres grupos específicos: madres/ padres estudiantes, población LGTBI y población de origen migrante. En este último caso, se solicita la generación de espacios para el intercambio cultural.

En resumen, podemos evaluar las disposiciones generales como positivas en tanto explicitan principios de diversidad y de acceso igualitario a la educación, pero la ausencia de disposiciones específicas al respecto del origen migrante de miembros de la comunidad dejaría dicho rasgo sin una regulación clara, lo que puede devenir en acciones discriminatorias por falta de herramientas administrativas a las cuales acudir.

\subsection{Nivel de gestión municipal}

Se revisaron en total 19 planes anuales de educación (Padem) de comunas de la provincia de Santiago, los cuales corresponden al $59 \%$ de las comunas de dicho territorio. Las comunas analizadas son aquellas que publican su Padem en sitio web o que enviaron el documento luego de realizar solicitud de información vía transparencia.

Con respecto a los planes anuales de educación municipal, se observan situaciones dispares pudiendo identificar tres tipos de comunas relacionadas con el trabajo educativo 
y la población migrante: municipios que incluyen estrategias explícitas de trabajo con dicha población, que denominaremos municipios integradores; municipios que no tienen referencia a trabajo con esa población; y municipios que tienen un foco en fortalecimiento de identidad nacional/local, que denominaremos municipios tradicionales, puesto que trabajan con una lógica asimilacionista donde lo que prima dentro de las directrices que guían el proceso de escolarización es la internalización de los elementos culturales de la cultura del país, asumiéndolas de modo homogéneo (Muñoz, 2000). Cabe destacar que este tipo de municipios tiene presencia de elementos culturales de corte rural, lo que podría explicar además la importancia de la generación de una identidad nacional pensada de modo homogéneo desde dichas tradiciones. Lo anterior se sistematiza en la Tabla 1:

Tabla 1

Presencia de estrategias NNA de origen migrante en Padem

\section{Categorización}

Municipios integradores

Municipios tradicionales

Municipios sin referencias
Municipios

Santiago, Recoleta, Estación Central, Quilicura

Lo Barnechea, Maipú

Peñalolén, La Pintana, Cerro Navia, Quinta Normal, Vitacura, La Cisterna, La Florida, La

Reina, Providencia, Las Condes, Pedro Aguirre

Cerda, Lo Espejo, Huechuraba

Fuente: Elaboración propia con base en documentos oficiales Padem.

Existe una coincidencia entre aquellos municipios que elaboran planes con respecto a la población migrante con los datos que desde la investigación han revelado sobre el comportamiento de la inserción de la población extranjera. Las principales acciones que estos municipios integran en su plan anual tienen que ver con la generación de un diagnóstico -que no es el mismo en todos los casos- de la población migrante que atienden en sus escuelas, considerando dimensiones tales como su nacionalidad, si su situación migratoria está regularizada o no, y la proporción existente en distintas escuelas dentro de la comuna. El resumen de la información encontrada se sistematiza en la tabla 2: 
Tabla 2

Porcentaje de migrantes presentes en municipios con diagnóstico

\begin{tabular}{cllcc}
\hline & Santiago & Recoleta & $\begin{array}{l}\text { Estación } \\
\text { Central }\end{array}$ & Quilicura \\
\hline $\begin{array}{c}\text { Porcentaje de } \\
\text { población migrante }\end{array}$ & $8.9 \%$ & $10.4 \%$ & $9.3 \%$ & $4.3 \%$ \\
$\begin{array}{c}\text { Principales } \\
\text { nacionalidades (en } \\
\text { orden jerárquico) } \\
\begin{array}{c}\text { Porcentaje de } \\
\text { población migrante } \\
\text { en situación irregular }\end{array}\end{array}$ & $\begin{array}{l}\text { Peruanos, } \\
\text { colombianos, }\end{array}$ & $\begin{array}{l}\text { Peruanos y } \\
\text { ecolombianos }\end{array}$ & $\begin{array}{c}\text { Sin } \\
\text { información }\end{array}$ & $\begin{array}{c}\text { Haitianos, } \\
\text { peruanos, } \\
\text { colombianos. }\end{array}$ \\
\hline
\end{tabular}

Fuente: Elaboración propia con base en documentos oficiales Padem.

Se evalúa como una acción integradora el diagnóstico, a pesar de ser una acción inicial y que no necesariamente incorpora una intervención directa en pro de la mejora de NNA de origen migrante, debido a que la inclusión en el plan anual de trabajo implica el reconocimiento de la diversidad cultural como una variable por considerar en la planificación del trabajo pedagógico, generando visibilidad del fenómeno migrante y valorando su relevancia en la planeación del trabajo del municipio.

Junto al diagnóstico, los municipios nombrados como integradores declaran la importancia de incluir acciones en el ámbito de la convivencia considerando la diversidad cultural. En caso de la comuna de Santiago, se incluye, asimismo, la intervención explícita para facilitar la regulación de situación migratoria de sus estudiantes.

Cabe señalar que existen comunas que trabajan dentro del Padem la dimensión interculturalidad, pudiendo entonces ser asociada de manera indirecta con la problemática migrante; estas corresponden a los municipios de Cerro Navia, La Florida, La Reina. Dichas comunas explicitan la importancia de la interculturalidad para el enriquecimiento de los procesos educativos en función de la valoración de la diversidad, pero no trabajan la migración como categoría relevante al respecto.

En el caso de los municipios tradicionales, sus planes de educación enfatizan acciones con el desarrollo de la identidad local, lo que orienta dichas acciones a la preservación de sus tradiciones. Cabe destacar que en los dos casos observados, se trata de municipios donde aún se observan consideraciones a la divulgación y promoción de una cultura rural en al menos parte del territorio de la comuna, lo que explica la importancia identitaria relativa que adquieren dichos elementos.

Cabe señalar el caso de la comuna de Quilicura que, a partir de las acciones realizadas por la Oficina de Atención a Migrantes y Refugiados en la sensibilización de 
problemáticas de dicha población, se ha generado una unidad de educación intercultural dentro del equipo del Departamento de Educación. Dicha unidad ha gestionado la contratación de un profesor de origen haitiano que pueda operar como mediador intercultural intercultural lingüístico con dicha población, en una iniciativa que aparece como única dentro de los municipios estudiados.

\section{Consideraciones finales}

Se hace necesario reconocer las limitaciones del presente estudio, las cuales tienen que ver con lo acotado de los dispositivos estudiados y los municipios representados. Esta última situación está mediada a su vez por el acceso a la información de los Padem y su publicación vía web. Una primera consideración, entonces, es la relevancia social que tiene la proyección de nuevos estudios que permitan realizar seguimiento a la política educativa asociada a la incorporación del estudiantado de origen migrante, aumentando la cobertura territorial y administrativa de los casos por considerar dentro de este.

A partir de los datos recopilados, se puede observar que a nivel macro, tanto la constitución vigente como la Ley General de Educación, resguarda el derecho a la educación de NNA de origen migrante al definir la escolaridad obligatoria y de acceso universal a lo largo del ciclo de escolarización. Asimismo, cabe considerar que dicha ley considera la diversidad cultural como parte de los principios básicos que sustentan el sistema. Sin embargo, estas declaraciones base contrastan con la ausencia de instrumentos que permitan de manera clara asegurar que la vivencia escolar de NNA de origen migrante pueda describirse de dicha manera.

También es importante considerar que declaraciones, tales como la no discriminación o el cuidado en la evaluación y promoción transparente del alumnado, no son necesariamente aplicadas en el caso de estudiantes de origen migrante. Existen antecedentes de que niños, niñas y adolescentes han sido sometidos a prácticas discriminatorias, tales como la negación de las matrículas en establecimientos, soportar tratos vejatorios al interior de establecimientos por parte de miembros de las comunidades educativas y que existen problemas en la certificación de los cursos aprobados debido a la ausencia de normativas claras al respecto y a la dependencia de las acciones del sistema al requisito de regulación de la situación migratoria (Olea, 2013; Pavez, 2012, 2013) .

Una de las principales conclusiones del presente trabajo tiene que ver con la baja presencia que tienen los principios de interculturalidad, diversidad y no discriminación 
presentes en dispositivos como la Ley General de la Educación o dispositivos técnico pedagógicos ministeriales como la política de convivencia escolar. De los 18 planes de educación municipal, solo tres de ellos (que representan un $17 \%$ ) tienen algún tipo acción educativa en relación con estudiantes extranjeros. El hecho de que no haya referencias a dicho grupo o que exista una intencionalidad de tipo tradicional nacionalista en el caso de los municipios restantes nos da cuenta de la baja concreción de dichos principios, así como de la necesidad de gestión, formación y generación de instrumentos que permitan transitar a modos de educación más inclusivos.

Asimismo, el hecho de que los planes de trabajo existentes estén focalizados en diagnóstico de la situación y la facilitación de la regulación de situación migratoria hace pensar en la necesidad de fortalecer dichas posturas, evitando el riesgo de configurar y estigmatizar estudiantes desde una postura deficitaria, haciendo que las intervenciones sean solo compensatorias y desde una postura paternalista.

Cuando se toman en cuenta los planes anuales de educación en las comunas, nos damos cuenta que existe una relación entre aquellas comunas que desde los estudios migratorios han indicado una presencia importante de migrantes -sea en número o en historicidad del fenómeno- lo que hace pensar de la predominancia de un enfoque reactivo a las necesidades por sobre la consideración de la interculturalidad como un principio de los procesos educativos en términos transversales, tal y como lo enuncia la Ley General de Educación. Asimismo, es un síntoma de la falta de operacionalización y regulación que tienen las disposiciones legales, existiendo importantes vacíos administrativos y procedimentales para llevarse a cabo y que afectan diversos actores involucrados. La baja presencia del fenómeno del estudiantado de origen extranjero y los desafíos que implica para la educación en los distintos dispositivos reguladores del proceso educativo conlleva dejar al arbitrio de voluntades personales y específicas -sea de establecimientos o de personas dentro de estos- la posibilidad de educación para estos niños, niñas y adolescentes.

Si bien es valorable que los Padem se ajusten a problemáticas emergentes, también es necesario considerar que dicho enfoque puede estigmatizar la presencia de estudiantes extranjeros dentro de su dimensión problemática, minimizando los aportes que tiene la diversidad cultural dentro de la experiencia educativa, ya sea para su abordaje dentro de los diseños didácticos de asignaturas, como de la construcción de culturas educativas que permitan abordar aprendizajes de tipo sociovalórico, tales 
como el respeto, la no discriminación, el diálogo o la construcción de una cultura de paz, todos ellos sugeridos por instancias supranacionales, tales como la Unesco.

Cabe considerar que este tipo de problemáticas heredan falencias que tienen los marcos reguladores tanto de la situación migratoria -ley de extranjería- y del sistema educativo en su globalidad. En el caso de la educación, este tipo de situaciones tienen que ver con la borradura de las diferencias identitarias sin considerar estas como insumo para los procesos de aprendizaje, la folklorización de las identidades distintas a la chilena o la consideración de la migración como un problema fundamentalmente administrativo, y con lógicas de transitoriedad.

La estructura institucional de la escuela, que desde sus inicios ha trabajado en función de la construcción de identidades nacionales, tiene lógicas tradicionales de su funcionamiento que están en torno a la homogeneidad cultural, considerando esta tanto como meta y como condición de funcionamiento. Esta tarea se lleva a cabo a partir de la generación de itinerarios que impelen a los NNA a internalizar elementos culturales tales como lenguaje, normas, principios valóricos y contenidos específicos de la cultura nacional.

Dichos rasgos pueden ayudar a explicar la falta de trabajo formativo en torno a la diversidad cultural, elemento que podría mediar al fenómeno reciente del aumento de la tasa de alumnado migrante. Un área privilegiada que debe tomarse en cuenta en este sentido son los programas de estudio de niños, niñas y adolescentes.

Según las observaciones 11 y 13 del Comité de derechos económicos, sociales y culturales que explícita las características que debiese tener la educación, podríamos considerar que las dimensiones de accesibilidad y disponibilidad estarían siendo satisfechas. Sin embargo, tanto la no consideración de la diversidad cultural en torno a temáticas tales como formación de identidad o reconocimiento de saberes al interior de los procesos educativos, ya sea en forma de estrategias o normativas que regulan la educación, como la falta de facilidades para aquellos niños, niñas y adolescentes en situación irregular para el reconocimiento de sus aprendizajes y avances curriculares, nos da antecedentes para considerar que los procesos de escolarización tienen falencias en la adaptabilidad a las necesidades que la población de origen extranjero pudiese tener lo que redunda en fallas con respecto a la aceptabilidad del proceso educativo.

Las principales recomendaciones para enfrentar la situación descrita a lo largo del presente trabajo tienen relación con la generación de dispositivos explícitos que permitan orientar la labor del sistema educativo para evitar la discriminación y menoscabo en el ejercicio de sus derechos de niños, niñas y adolescentes de origen migrante. Asimismo, 
generar una institucionalidad centralizada que se hiciera cargo de la inserción de niños, niñas y adolescentes en el sistema educacional chileno, asegurando su inclusión sin problemas en éste. Es necesario definir claramente las dimensiones que se deben cautelar en la inclusión del estudiantado de origen migrante y las garantías que debe dar el sistema educativo, para aumentar la exigibilidad de dichos derechos. Una posibilidad para el abordaje de este desafío podría ser ampliar la Ley 20422 que se refiere a la discriminación en situación de discapacidad a todo tipo de discriminación incluyendo en estas las derivadas de la dimensión de identidad, asumiendo en esta los aportes propios de la ley contra la discriminación.

A su vez, se hace necesario generar procedimientos claros y obligatorios para las escuelas -independientemente de su tipo de administración- frente a este tipo de situaciones. Dentro de estos es importante que la gestión de la documentación de la historia escolar de niños, niñas y adolescentes no dependa de recursos, criterios y procedimientos específicos de unidades educativas determinadas. Cabe destacar que hay situaciones, como el idioma o la existencia de asignaturas y estructuras formativas, que no tienen símil en nuestro país, pues generan obstáculos importantes para dar respuesta a la matrícula de estudiantes con estudios en el extranjero. Asimismo, en los casos que se considere pertinente el que estudiantes dieran algún tipo de evaluación con consecuencias administrativo-pedagógicas, tales como homologación de notas de asignaturas o dar un juicio taxativo con respecto al nivel al que pertenecería cada NNA, se hace necesario que existan procedimientos y evaluaciones transparentes y que no impliquen discriminación, tal como lo plantea nuestra Ley General de Educación y las normativas emitidas desde el gobierno central al respecto.

La necesidad de operacionalizar de mejor manera la concreción de las disposiciones generales del sistema educativo implica también la realización de un mejor diagnóstico con respecto a la realidad del estudiantado de origen migrante. Se destacan de manera específica dos ámbitos por trabajar; el primero, es necesario pensar más allá de las disposiciones formales de ciudadanía y nación, puesto que los diagnósticos se refieren a estudiantes nacidos fuera del país; un niño o niña nacido en Chile se considera para las estadísticas como chileno, sin considerar las características del entorno cultural de su crianza y socialización primaria. El segundo ámbito tiene relación con la generación de instrumentos que permitan la comparación de realidades entre las unidades territoriales así como entre periodos de tiempo de modo de mejorar la gestión administrativa - pedagógica. 
Sin embargo, cualquier intervención de mejora resulta incompleta si no se incluye una reflexión profunda acerca de cuál va ser la postura formativa de las escuelas con respecto a problemáticas, tales como la identidad cultural, identidad nacional y su impacto en la configuración de metas de aprendizaje, para poder diseñar, ejecutar y evaluar propuestas de formación. Por ejemplo es necesario responderse: ¿es necesario que en el mundo actual los NNA aprendan solo historia y geografía asociada a la nación? ¿Los niños de origen extranjero tienen que preservar solo su cultura de origen? ¿De qué manera se debieran incluir otras culturas a la escuela? ¿Cuáles son los aprendizajes necesarios para lograr una formación que equilibre el respeto por la diversidad cultural y la integración que requiere la cohesión social? En suma, ¿cómo generar una escuela pertinente a un escenario social globalizado?

Las anteriores interrogantes son fundamentales para dar un paso más allá en el abordaje de esta problemática, asumiendo las consideraciones pedagógicas que tiene, no centrando los esfuerzos para la instalación de un proceso educativo que cautele elementos formales y administrativos dejando de lado los fines y sentidos de la educación. En ese sentido, cabe destacar que damos los primeros pasos de un camino que va desde reconocer la diversidad como parte del entorno, sin borrar o negar la diferencia, hasta llegar a la inclusión de dichos rasgos de diversidad como un insumo para el proceso educativo, aumentando la pertinencia e impacto de la experiencia escolar.

\section{Referencias}

Agar, Lorenzo. (2012). Migraciones posmodernas en Chile: Reflexiones sobre cohesión social y pluralidad cultural. Palobra, (12), 106-127.

Banco Mundial. (2015). Migración Neta. Recuperado de http://datos.bancomundial.org/indicador/SM.POP.NETM

Chile, Departamento Extranjería y Migración. (28 de enero, 2003). Oficio Circular 1179 Recuperado de http://www.extranjeria.gob.cl/filesapp/Oficio\%20Circular\%201179.pdf

Chile, Instituto Nacional de Derechos Humanos. (2012). Compilación de recomendaciones al Estado de Chile de los órganos del sistema universal y del sistema interamericano de Derechos Humanos. Santiago: Instituto Nacional de Derechos Humanos.

Chile, Ministerio de Educación. (26 de junio, 2009). Decreto 254: Modifica Decreto Supremo 220 del Ministerio de Educación que establece los Objetivos Fundamentales y Contenidos Mínimos Obligatorios de la Educación Media. Santiago, Chile: Biblioteca del Congreso Nacional de Chile. 
Chile, Ministerio de Educación. (2011). Decreto 439: Establece bases curriculares para la educación básica en asignaturas que se indica. Santiago: Biblioteca del Congreso Nacional de Chile.

Chile, Ministerio de Educación. (17 de septiembre, 2011). Ley 20.370 Ley General de Educación. Santiago, Chile: Biblioteca del Congreso Nacional de Chile.

Chile, Ministerio de Educación. (8 de septiembre, 2011). Ley 20536 Ley de Violencia Escolar. Santiago, Chile: Biblioteca del Congreso Nacional de Chile.

Chile, Ministerio de Educación. (2014). Otros Indicadores de Calidad. Santiago: Unidad de $\begin{array}{llll}\text { Curriculum } & y & \text { Evaluación. } & \text { Recuperado }\end{array}$ http://www.curriculumenlineamineduc.cl/605/articles-33859 recurso 93.pdf

Chile, Ministerio de Educación. (2015). Política Nacional de Convivencia Escolar 2015-2018. Recuperado de http://www.mineduc.cl/wp-content/uploads/sites/19/2015/12/politicanoviembre-definitiva.pdf

Lagos, Víctor y Rojas, Óscar. (2014). Derechos de los Migrantes y Refugiados. En Informe Anual sobre Derechos Humanos en Chile 2014 (pp. 333-376). Santiago: Ediciones Universidad Diego Portales.

López, Néstor. (2014). Mercado o Garantía de Derechos: Modelos en debate para la educación escolar en Chile. Santiago de Chile: Unicef - IIPE/Unesco.

Mejía, Julio. (2004). Sobre investigación cualitativa. Nuevos conceptos y campos de desarrollo. Investigaciones Sociales, 8(13), 277-299.

Muñoz, Antonio. (2000). Hacia una educación intercultural: enfoques y modelos. Encuentros sobre educación, 1, 81-108.

Norambuena, Carmen. (2013). Amenazas sin fronteras: nuevas periferias en la circulación de personas. Cuadernos de Historia, (39), 147-168.

OEA. (1988). Protocolo Adicional a la Convención Americana sobre Derechos Humanos en materia de Derechos Económicos Sociales y Culturales, Protocolo de San Salvador. Recuperado de http://repositorio.dpe.gob.ec/handle/39000/607

Olea, Helena. (2013). Derechos Humanos de migrantes y refugiado. Análisis del proyecto de Ley de migración y extranjería. En Informe Anual sobre Derechos Humanos en Chile 2013 (pp. 123-162). Santiago: Ediciones Universidad Diego Portales.

Pavez, Izkra. (2012). Inmigración y racismo: experiencias de la niñez peruana en Santiago de Chile. Si somos americanos. Revista de estudios transfronterizos, 12(1), 75-99.

Pavez, Izkra. (2013). Los significados de "ser niña y niño migrante": conceptualizaciones desde la infancia peruana en Chile. Polis, Revista Latinoamericana, 12(35), 183-210.

Tomasvski, Katarina. (2004). Los derechos económicos, sociales y culturales: El derecho a la educación. Informe presentado a la Comisión de Derechos Humanos en su 60avo periodo de sesiones. Recuperado de www.oei.es/decada/portadas/G0410331.pdf 